\title{
Von Neumann's Comparison Method for Random Sampling from the Normal and Other Distributions*
}

\author{
By George E. Forsythe**
}

\begin{abstract}
The author presents a generalization he worked out in 1950 of von Neumann's method of generating random samples from the exponential distribution by comparisons of uniform random numbers on $(0,1)$. It is shown how to generate samples from any distribution whose probability density function is piecewise both absolutely continuous and monotonic on $(-\infty, \infty)$. A special case delivers normal deviates at an average cost of only 4.036 uniform deviates each. This seems more efficient than the Center-Tail method of Dieter and Ahrens, which uses a related, but different, method of generalizing the von Neumann idea to the normal distribution.
\end{abstract}

1. Introduction. In the summer of 1949 , at the Institute for Numerical Analysis on the campus of the University of California, Los Angeles, John von Neumann [3] lectured on various aspects of generating pseudorandom numbers and variables. At the end, he presented an ingenious method for generating a sample from an exponential distribution, based solely on comparisons of uniform deviates. In his last sentence, he commented that his "method could be modified to yield a distribution satisfying any first-order differential equation".

In 1949 or 1950, I wrote some notes about what I assumed von Neumann had in mind, but I do not recall ever discussing the matter with him. This belated polishing and publication of those notes is stimulated by papers by Ahrens and Dieter [1], [2] in which several related algorithms are studied, and by a personal discussion with the authors on how the von Neumann idea can be extended.

In Section 2, the general method is presented, and in Section 3, its efficiency is analyzed. In Sections 4 and 5 , it is shown how the exponential and normal distributions show up as special cases. In Section 6, the method for a normal distribution is compared with the Center-Tail method of [1] and [2]. In Section 7, possible generalizations are mentioned.

Received April 27, 1972.

AMS 1969 subject classifications. Primary 6515, 6020.

Key words and phrases. Random variables, random deviates, normal, sample, von Neumann, comparison, center, tail, Gaussian, distribution.

* This research was supported in part by the Office of Naval Research under contracts N-0001467-A-0112-0057 (NR 044-402) and N-00014-67-A-0112-0029 (NR 044-211), and by the National Science Foundation under grant GJ-29988X.

** This paper was submitted posthumously. We mourn the loss of our friend and pioneer in the field of computer science.

The author's research was in progress at the time of his death. He had discussed these matters with his colleagues J. H. Ahrens and U. Dieter, and had also interested his former student R. Brent in the work. It is expected that a future issue of this journal will contain some paper (or papers) by this group with extensions of Forsythe's work.

Copyright (C) 1972, American Mathematical Society 
Although this introduction has emphasized historical matters, the method of Section 6 is a good one, and is competitive with the best known methods for generating normal deviates.

I thank both Professors Ahrens and Dieter for their careful criticism of a first draft of this paper.

2. The General Algorithm. Let $f(x)>0$ be defined for all $x \geqq 0$ and satisfy the first-order linear differential equation

$$
f^{\prime}(x)+b(x) f(x)=0 \quad(0 \leqq x<\infty),
$$

where $b(x) \geqq 0$. Let

$$
B(x)=\int_{0}^{x} b(t) d t
$$

and assume that

$$
C=\int_{0}^{\infty} e^{-B(x)} d x<\infty
$$

Then

$$
f(x)=C^{-1} e^{-B(x)}
$$

is the unique solution of (1) with $\int_{0}^{\infty} f(x) d x=1$, and hence $f$ is the probability density distribution of a nonnegative random variable.

Suppose we have a supply of independent random variables $u$ with a uniform distribution on $[0,1)$, and that we wish to generate a random variable $y$ with the density distribution $f(x)$. Here is one way to proceed.

We first prepare three tables of constants $\left\{q_{k}\right\},\left\{r_{k}\right\},\left\{d_{k}\right\}$ for $k=0,1, \cdots, K$, as follows. ( $K$ is defined below.) Let $q_{0}=0$. For each $k=1,2, \cdots, K$, pick $q_{k}$ as large as possible, subject to the two constraints

$$
\begin{aligned}
q_{k}-q_{k-1} & \leqq 1, \\
B\left(q_{k}\right)-B\left(q_{k-1}\right) & \leqq 1 .
\end{aligned}
$$

Next, compute

$$
r_{k}=\int_{0}^{a_{k}} f(x) d x \quad(k=0,1, \cdots, K)
$$

Here $K$ is chosen as the least index such that $r_{K}$ exceeds the largest representable number less than 1. ( $K$ may be chosen smaller, if one sets $r_{K}=1$, and if one is willing to truncate the generated variable by reducing any value above $q_{K}$ to the interval $\left[q_{K-1}, q_{K}\right)$.) Finally, compute

$$
d_{k}=q_{k}-q_{k-1} \quad(k=1,2, \cdots, K) .
$$

For simplicity, we define the functions

$$
G_{k}(x)=B\left(q_{k-1}+x\right)-B\left(q_{k-1}\right) \quad(k=1,2, \cdots, K) .
$$

*** Editorial comment: The referees thought condition (5) to be superfluous. 
Now, we present the algorithm. Steps 1 to 3 determine which interval $\left[q_{k-1}, q_{k}\right)$ the variable $y$ will belong to. Steps 4 to 11 determine the value of $y$ within that interval.

1. [Begin choice of interval.] Set $k \leftarrow 1$. Generate a uniform deviate $u$.

2. [Test.] If $u \leqq r_{k}$, go to step 4 .

3. [Increase interval.] If $u>r_{k}$, set $k \leftarrow k+1$ and go back to step 2 .

4. [Begin computation of $y$ in the selected interval.] Generate another uniform deviate $u$ and set $w \leftarrow u d_{k}$.

5. Set $t \leftarrow G_{k}(w)$.

6. Generate another uniform deviate $u^{*}$.

7. [Test.] If $u^{*} \geqq t$, go to step 11 .

8. [Trial continues.] If $u^{*}<t$, generate another uniform deviate $u$.

9. [Test.] If $u<u^{*}$, set $t \leftarrow u$ and go back to step 6 .

10. [Reject the trial.] If $u \geqq u^{*}$, go back to step 4 .

11. [Finish.] Return $y \leftarrow q_{k-1}+w$ as the sample variable.

We now show that the above algorithm works as claimed. Since we assume that each $u<1$, the test in step 2 must be passed when $k=K$, if not sooner. Hence, an interval $\left[q_{k-1}, q_{k}\right)$ is selected, and the values of $r_{k}$ were chosen to make the probabilities of choosing the various intervals correct.

Fix $k$. The remainder of the algorithm can be described as follows: First, a random number $w$ is selected uniformly from the interval $0 \leqq w \leqq d_{k}$. Then the algorithm continues to generate independent uniform deviates $u_{i}$ from $[0,1)$ until the least $n$ is found with

$$
\begin{array}{rlrl}
u_{2} & \geqq G_{k}(w) & (n=1), & \text { or } \\
u_{n+1} \geqq u_{n}<u_{n-1}<\cdots<u_{3}<u_{2}<G_{k}(w) & (n \geqq 2) . &
\end{array}
$$

With probability 1 , such an $n$ will be found, as will be shown. If $n$ is odd, we return $y \leftarrow q_{k-1}+w$. If $n$ is even, we reject $w$ and all the $u$, choose a new $w$, and repeat.

We now determine the probability $P(k, w)$ that one $w$ determined in step 4 will be accepted without returning to step 4 . Let $E_{1}(k, w)$ be the universe of all events. For $n=2,3, \cdots$, let $E_{n}(k, w)$ be the event

$$
u_{n}<u_{n-1}<\cdots<u_{3}<u_{2}<G_{k}(w) .
$$

Then the probability of $E_{n}(k, w)$ is given by

$$
\begin{array}{rlrl}
\operatorname{Prob}\left\{E_{u}(k, w)\right\} & =1 & (n=1) \\
& =\int_{0}^{G_{k}(w)} d x_{2} & (n=2) \\
& =\int_{0}^{G_{k}(w)} d x_{2} \int_{0}^{x_{2}} d x_{3} \cdots \int_{0}^{x_{n-1}} d x_{n} & & (n \geqq 3) \\
& =G_{k}(w)^{n-1} /(n-1) ! & & (\text { all } n) .
\end{array}
$$

The occurrence of $(10)$ is the conjunction of $E_{n}(k, w)$ and not- $E_{n+1}(k, w)$. Since $E_{n+1}(k, w)$ implies $E_{n}(k, w)$, the probability that (10) occurs for a given $n$ and $w$ is

$$
\operatorname{Prob}\left\{E_{n}(k, w) \text { and not- } E_{n+1}(k, w)\right\}=\frac{G_{k}(w)^{n-1}}{(n-1) !}-\frac{G_{k}(w)^{n}}{n !} .
$$


Summing over all odd $n$, we see that

$$
P(k, w)=\sum_{\text {odd } n}\left[\frac{G_{k}(w)^{n-1}}{(n-1) !}-\frac{G_{k}(w)^{n}}{n !}\right]=e^{-G_{k}(w)} .
$$

Since $w \leqq d_{k}$, we have

$$
G_{k}(w) \leqq G_{k}\left(d_{k}\right)=B\left(q_{k}\right)-B\left(q_{k-1}\right) \leqq 1,
$$

whence

$$
P(k, w) \geqq e^{-1}, \quad \text { for all } k \text { and } w .
$$

Now $d_{k}^{-1} d \xi$ is the probability that $w$ is selected in the interval $\xi \leqq w \leqq \xi+d \xi$. Combining this with (12), we see that the probability that $\xi \leqq w \leqq \xi+d \xi$ and that $w$ is accepted is given by

$$
\operatorname{Prob}\{\xi \leqq w \leqq \xi+d \xi \text { and } w \text { is accepted }\}=e^{-G_{k}(w)} d \xi / d_{k} .
$$

Corresponding to an accepted $w$, we return $y=q_{k-1}+w$ as the sample variable. Hence, from (14), the probability that $y$ is in the range $x \leqq y \leqq x+d x$, for given $k$, is

$$
\begin{aligned}
\frac{1}{d_{k}} e^{-G_{k}\left(x-q_{k-1}\right)} d x & =\frac{1}{d_{k}} e^{-B(x)+B\left(q_{k-1}\right)} d x, \\
& =\frac{1}{d_{k}} C e^{B\left(q_{k}-1\right)} C^{-1} e^{-B(x)} d x \\
& =\frac{1}{d_{k} f\left(q_{k-1}\right)} f(x) d x,
\end{aligned}
$$

That is,

$$
\operatorname{Prob}\{x \leqq y \leqq x+d x \text { and } y \text { is accepted }\}=\frac{f(x) d x}{d_{k} f\left(q_{k-1}\right)} .
$$

Since this is proportional to $f(x) d x$, we see that any accepted $y$ has the desired probability density distribution within the interval $\left[q_{k-1}, q_{k}\right)$. Since, from (13), the probability of an infinite loop back to step 4 is zero, the second half of the algorithm terminates with probability 1 . This concludes the demonstration that the algorithm works as claimed.

3. Efficiency of the Algorithm. For a general function $b$, I shall derive a representation for the expected number of uniformly distributed random variables $u$ that must be used to generate one variable $y$ with the probability density proportional to $f(x)$. A similar derivation is given in [2].

The preliminary game to select $k$-steps 1 to 3 of the algorithm-requires one $u$.

The rest of the algorithm is different for each $k$, and we shall first determine the expected number $N(k)$ of steps to determine $y$. To do this, we shall first assume that $k$ is fixed and that $w$ has been picked in the interval $0 \leqq w \leqq d_{k}$. Define $E_{n}(k, w)$ as in Section 2, and introduce the abbreviations

$$
e_{n}=e_{n}(k, w)=\operatorname{Prob}\left\{E_{n}(k, w)\right\} \quad(n=1,2, \cdots)
$$

and 


$$
g=G_{k}(w) \text {. }
$$

Then, as in Section 2, we have the following expression for the probability $P(k, w)$ of accepting $w$ without returning to step 4:

$$
P(k, w)=\left(e_{1}-e_{2}\right)+\left(e_{3}-e_{4}\right)+\left(e_{5}-e_{6}\right)+\cdots .
$$

Moreover, given $k$ and $w$ and given that $w$ is accepted, the expected number of uniform deviates $u$ needed will be

$$
\begin{aligned}
m_{a}(k, w) & =P(k, w)^{-1}\left[2\left(e_{1}-e_{2}\right)+4\left(e_{3}-e_{4}\right)+6\left(e_{5}-e_{6}\right)+\cdots\right] \\
& =\frac{1}{P(k, w)} \sum_{\text {odd } n}\left[\frac{g^{n-1}}{(n-1) !}-\frac{g^{n}}{n !}\right](n+1) .
\end{aligned}
$$

Similarly, the probability $1-P(k, w)$ that $w$ is rejected is given by

$$
1-P(k, w)=\left(e_{2}-e_{3}\right)+\left(e_{4}-e_{5}\right)+\left(e_{6}-e_{7}\right)+\cdots .
$$

Moreover, given $k$ and $w$ and that $w$ is rejected, the expected number of uniform deviates $u$ needed is

$$
\begin{aligned}
m_{r}(k, w) & =[1-P(k, w)]^{-1}\left[3\left(e_{2}-e_{3}\right)+5\left(e_{4}-e_{5}\right)+7\left(e_{6}-e_{7}\right)+\cdots\right] \\
& =\frac{1}{1-P(k, w)} \sum_{\text {even } n ; n \geqq 2}\left[\frac{g^{n-1}}{(n-1) !}-\frac{g^{n}}{n !}\right](n+1) .
\end{aligned}
$$

Now, if a $w$ is rejected, the algorithm returns to step 4, a new $w$ is picked, and the process repeats. Let $M(k, w)$ be the expected number of uniform deviates selected until a $y$ is finally selected, given a fixed $k$ and an initially chosen $w$. Then $N(k)$ is the average of $M(k, w)$ over all $w$ uniformly distributed on $0 \leqq w \leqq d_{k}$.

We have

$$
M(k, w)=P(k, w) m_{a}(k, w)+[1-P(k, w)]\left[m_{r}(k, w)+N(k)\right],
$$

since, in case $w$ is rejected, the whole process is repeated. Using the expressions (18) and (19) for $m_{a}(k, w)$ and $m_{r}(k, w)$, we get from (20) that

$$
\begin{aligned}
M(k, w) & =\sum_{n=1}^{\infty}\left[\frac{g^{n-1}}{(n-1) !}-\frac{g^{n}}{n !}\right](n+1)+[1-P(k, w)] N(k) \\
& =1+e^{g}+[1-P(k, w)] N(k),
\end{aligned}
$$

or

$$
M(k, w)=1+e^{G_{k}(w)}+[1-P(k, w)] N(k) .
$$

Averaging (21) for $0 \leqq w \leqq d_{k}$, and using (12), we find that

$$
N(k)=1+\frac{1}{d_{k}} \int_{0}^{d_{k}} e^{G_{k}(w)} d w+N(k)\left[1-\frac{1}{d_{k}} \int_{0}^{d_{k}} e^{-G_{k}(w)} d w\right] .
$$

Solving for $N(k)$, we get

$$
N(k)=\left(d_{k}+\int_{0}^{d_{k}} e^{G_{k}(w)} d w\right) / \int_{0}^{d_{k}} e^{-G_{k}(w)} d w
$$


Finally, the expected number $\bar{N}$ of uniform deviates drawn in the main game until a $y$ is returned is the average of $N(k)$ over the intervals, weighted by the probabilities of selecting the various intervals. That is,

$$
\bar{N}=\sum_{k=1}^{\infty} N(k)\left[r_{k}-r_{k-1}\right] .
$$

If we make use of (4), (7), and (9) to express $\bar{\Gamma}$ in terms of $B(x)$, we obtain the ugly representation

$$
\begin{aligned}
\bar{N} & =\sum_{k=1}^{\infty} \frac{d_{k}+e^{-B\left(q_{k-1}\right)} \int_{q_{k-1}}^{q_{k}} e^{B(x)} d x}{e^{B\left(q_{k-1}\right)} \int_{q_{k-1}}^{q_{k}} e^{-B(x)} d x} \cdot \frac{\int_{q_{k-1}}^{q_{k}} e^{-B(x)} d x}{\int_{0}^{\infty} e^{-B(x)} d x} \\
& =\frac{1}{\int_{0}^{\infty} e^{-B(x)} d x} \sum_{k=1}^{\infty}\left[d_{k} e^{-B\left(q_{k-1}\right)}+e^{-2 B\left(q_{k-1}\right)} \int_{q_{k-1}}^{q_{k}} e^{B(x)} d x\right] .
\end{aligned}
$$

4. Special Case: Exponential Distribution. If $b(x)=1$ in (1), then $B(x)=x$ and $y(x)=e^{-x}$, corresponding to the exponential distribution treated in [3]. For the algorithm of Section 2, we have $q_{k}=k, d_{k}=1, r_{k}=1-e^{-k}$, and $G_{k}(x)=x$, for all $k$. Since $d_{k}$ and $G_{k}(x)$ are independent of $k$, steps 4 to 10 of the algorithm are the same for all $k$. They can therefore be carried out independently of steps 1 to 3 . By (12), the probability that a chosen $w$ is not accepted is $1-P(k, w)=1-e^{-w}$ (for all $k$ ), and the average value of $1-e^{-w}$ over $0 \leqq w \leqq 1$ is $e^{-1}$.

If the preliminary game of steps 1 to 3 were played, the interval $[k-1, k)$ would be selected with probability $r_{k}-r_{k-1}=e^{-(k-1)}-e^{-k}=e^{-k}(e-1)$, for $k=1$, $2, \cdots$. Thus the interval $[0,1)$ would be accepted with probability $1-e^{-1}$, and rejected with probability $e^{-1}$. For $k=1,2, \cdots$, if $[k-1, k)$ is rejected, then $[k$, $k+1)$ would be accepted with probability $1-e^{-1}$, and rejected with probability $e^{-1}$. Since the rejection ratio for each interval has the same value $e^{-1}$, which is the a priori probability of rejecting in the main game any $w$ selected in step 4, von Neumann could use the rejection of $w$ as the signal to change the interval from $[k-1, k)$ to $[k, k+1)$. Thus, the preliminary game of steps $1-3$ is unnecessary for the exponential distribution. This made von Neumann's game very elegant. I know of no comparable trick for general $b(x)$.

From (22) and (23), since $N(k)=\bar{N}$, we see that for the exponential distribution

$$
\bar{N}=\frac{1+(e-1)}{1-e^{-1}}=\frac{e}{1-e^{-\overline{1}}} \doteqdot 4.30026,
$$

as stated in [1]. There was an error in [3].

5. Special Case: Normal Distribution. If $b(x)=x$ in (1), then $B(x)=x^{2} / 2$ and $f(x)=(2 / \pi)^{1 / 2} e^{-x^{2} / 2}$, corresponding to the positive half of the normal distribution. For the algorithm of Section 2, we have

$$
q_{0}=0, \quad q_{1}=1, \cdots, \quad q_{k}=(2 k-1)^{1 / 2} \quad(k \geqq 2) .
$$

Hence, 
$d_{1}=1, \quad d_{2}=3^{1 / 2}-1, \cdots, \quad d_{k}=(2 k-1)^{1 / 2}-(2 k-3)^{1 / 2} \quad(k \geqq 2)$.

Also,

$$
G_{k}(x)=x^{2} / 2+q_{k-1} x \quad(k \geqq 1) .
$$

The values of $r_{k}$ must be computed from the probability integral. The table below gives 15 decimal values of $q_{k}, d_{k}, r_{k}$, and $N(k)$ for $k=1,2, \cdots, 36$, as computed in Fortran on Stanford's IBM 360/67 computer in double precision.

To generate normal deviates, one selects $K$ and prestores the values of $r_{k}, q_{k}$, and $d_{k}$ for $k=1,2, \cdots, K$. Then set $q_{0} \leftarrow 0$ and $d_{K} \leftarrow 1$. (The limit $K=12$ permits normal deviates up to \pm 5.0 to be generated, and the deviates will be truncated less than once in a million trials. A higher limit will decrease the probability of truncation.

As suggested in [2], one should start the algorithm with a preliminary determination of the sign of the normal deviate. We do this in steps N1-N3 of the following algorithm. At entry to step $\mathrm{N} 4, u$ is a uniform deviate on the interval $[0,1)$. The rest is the algorithm of Section 2, with the sign appended in the last step.

N1. [Begin choice of sign and interval.] Set $k \leftarrow 1$. Generate a uniform deviate $u$ on $[0,1)$. Set $u \leftarrow 2 u$.

N2. [Test for sign.] If $u<1$, set $s \leftarrow 1$ and go to step N4.

N3. If $u \geqq 1$, set $s \leftarrow-1$, and set $u \leftarrow u-1$.

N4. [Test for interval.] If $u \leqq r_{k}$, go to step N6.

N5. [Increase interval.] If $u>r_{k}$, set $k \leftarrow k+1$ and go back to step N4.

N6. [Begin generation of $|y|$ in the selected interval.] Generate another uniform deviate $u$ on $[0,1)$ and set $w \leftarrow u d_{k}$.

N7. Set $t \leftarrow G_{k}(w)$.

N8. Generate another uniform deviate $u^{*}$ on $[0,1)$.

N9. [Test.] If $u^{*} \geqq t$, go to step N13.

N10. [Trial continues.] If $u^{*}<t$, generate another uniform deviate $u$ on $[0,1)$.

N11. [Test.] If $u<u^{*}$, set $t \leftarrow u$ and go back to step N8.

N12. [Reject the trial.] If $u \geqq u^{*}$, go back to step N6.

N13. [Finish.] Return $y \leftarrow s\left(q_{k-1}+w\right)$ as the sample normal variable.

As in Section 3, we let $N(k)$ be the expected number of selections of uniform deviates in steps N4-N13, as a function of $k$. We have from (22):

$$
\begin{aligned}
& N(1)=\frac{1+\int_{0}^{1} e^{w^{2} / 2} d w}{\int_{0}^{1} e^{-w^{2} / 2} d w}, \\
& N(k)=\frac{d_{k}+e^{3 / 2-k} \int_{a_{k-1}}^{q_{k}} e^{w^{2} / 2} d w}{e^{k-3 / 2} \int_{a_{k-1}}^{q_{k}} e^{-w^{2} / 2} d w} \quad(k \geqq 2) .
\end{aligned}
$$

Numerical values of $N(k)$ are given in the table. Using the asymptotic formula

$$
\int_{x}^{x+h} e^{ \pm t^{2} / 2} d t \sim \pm\left[\frac{e^{ \pm x^{2} / 2}}{x}\left(1 \pm \frac{1}{x^{2}}\right)\right]_{x}^{x+h}, \quad \text { as } x \rightarrow \infty
$$

one can show that 


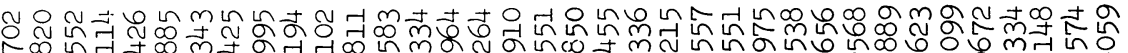
人on.

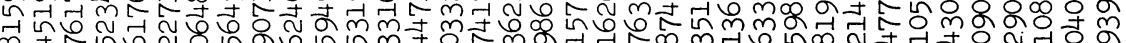

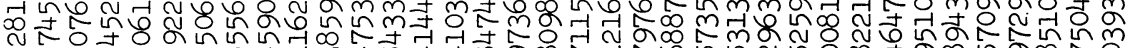

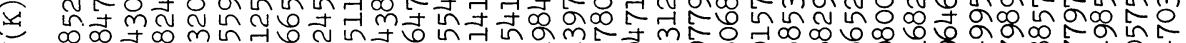
mp

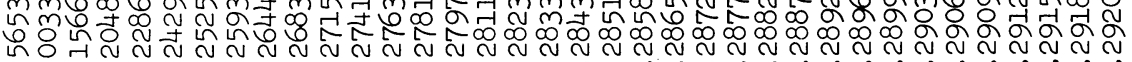

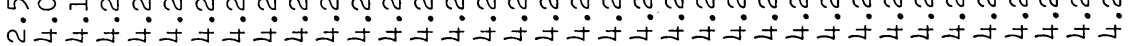
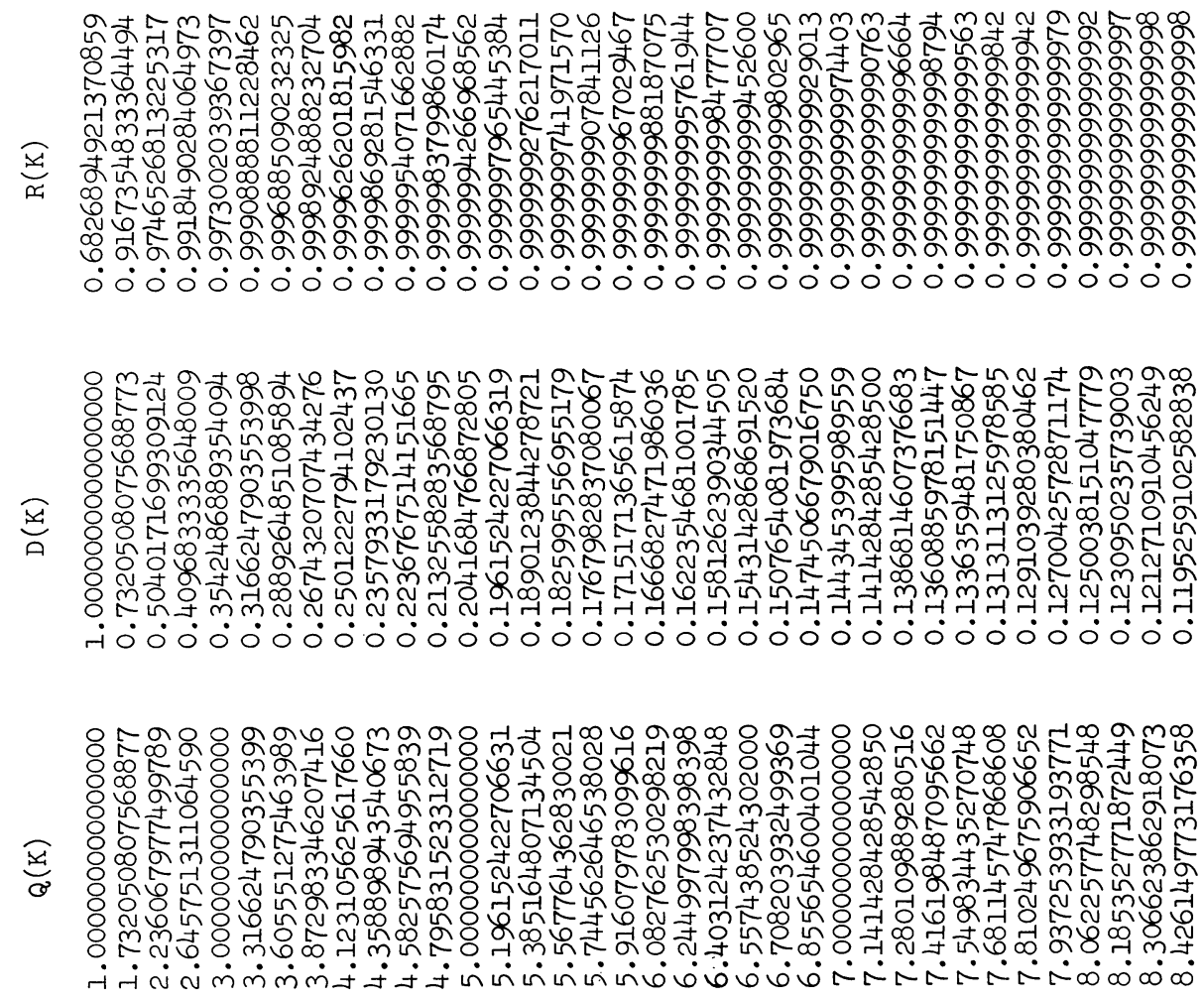


$$
\lim _{k \rightarrow \infty} N(k)=e /\left(1-e^{-1}\right) \doteqdot 4.30026 .
$$

Cf. (25). The equality (26) was written to me by U. Dieter.

I have used the same computer to establish that

$$
\bar{N}=\sum_{k=1}^{\infty} N(k)\left(r_{k}-r_{k-1}\right) \doteqdot 3.03585,
$$

so that the expected number of uniform deviates chosen in order to generate one normal deviate is $1+\bar{N} \doteqdot 4.03585$.

The correctness of this algorithm for generating normal deviates, as well as the value of $N$, have been confirmed in unpublished experiments by $\mathrm{A}$. I. Forsythe and independently by $\mathbf{J}$. H. Ahrens.

6. Comparison with the Center-Tail Method of Dieter and Ahrens. In [1], Dieter and Ahrens give a related but different modification of the von Neumann idea for the generation of normal deviates. There are only two intervals, the center and the tail, and the algorithms are quite different for the two. The expected number of uniform deviates needed is near 6.321, and computation of a square root is required in approximately 16 per cent of the cases.

The algorithm of Section 5 above requires no function call, but its main advantage over the Center-Tail method lies in requiring about two-thirds the number of uniform deviates. This should be reflected in a shorter average time of execution.

The Dieter-Ahrens algorithm for the center interval closely resembles my algorithm for each interval, and the proofs are very close to those given above. The big difference is that in [1] all variables $u_{i}$ have the cumulative distribution function $x^{2}(0 \leqq x \leqq 1)$, and the comparisons are of the form

$$
u_{n+1} \geqq u_{n}<u_{n-1}<u_{n-2}<\cdots<u_{3}<u_{2}<u_{1} .
$$

In contrast, in this paper all variables $u_{i}$ have uniform distributions and the comparisons take the form (for the principal case $k=1$ ):

$$
u_{n+1} \geqq u_{n}<u_{n-1}<u_{n-2}<\cdots<u_{3}<u_{2}<u_{1}^{2} / 2 .
$$

Changing the distribution function in [1] costs an extra uniform deviate and a comparison for each $u_{i}$, whereas forming $u_{1}^{2} / 2=G_{1}(w)$ in Section 5 is done only once for each chain of $u_{i}$ 's. Moreover, the fact that $u_{1}^{2} / 2$ is usually small means that, most of the time, $u_{2} \geqq u_{1}^{2} / 2$ and hence $u_{1}$ is accepted immediately. This contributes to keeping $N$ low in my algorithm. Finally, the use of $G_{k}(w)$ makes it possible to use the von Neumann technique in any interval in which $G_{k}(w)$ can be evaluated.

In a more recent manuscript [2], Dieter and Ahrens have improved their CenterTail method so that the comparisons are simpler and the expected number of uniform deviates needed is reduced to near 5.236. According to the authors, the improved Center-Tail method is still somewhat slower than my algorithm.

7. Further Generalizations. Let $f(x)(-\infty<x<\infty)$ be the probability density function of a random variable $F$. Under what conditions on $f$ could the von Neumann idea be applied to pick a sample from $F$ ? It is sufficient that the interval $(-\infty, \infty)$ be the union of a set of abutting intervals $I_{k}=\left[q_{k-1}, q_{k}\right](k=\cdots,-2,-1,0,1$, 
$2, \cdots)$ such that, in each closed interval $I_{k}$, either $f(x) \equiv 0$ or the following three conditions all hold: $f(x)>0, f$ is absolutely continuous, and $f$ is monotonic.

Then a preliminary game can be played to select an interval $I_{k}$. If $b(x)=$ $-f^{\prime}(x) / f(x) \geqq 0$ in $I_{k}$, the algorithm of Section 2 can be adapted to select a value in $I_{k}$ with a density distribution proportional to $f(x)$. (It may be necessary to subdivide $I_{k}$ so that (5) and (6) hold.)

If $b(x)<0$ in $I_{k}$, change $x$ to $-x$ and follow an analogous algorithm.

The main practical difficulties of the algorithm are these:

(a) One must evaluate various integrals like $\int_{a}^{x} f(t) d t$, in order to determine the parameters needed to pick the intervals $I_{k}$ during execution, and to evaluate the needed $r_{k}, q_{k}$, and $d_{k}$. These computations have to be done only once in designing the algorithm.

(b) One must evaluate $G_{k}(w)$ for arbitrary $w$ in $\left[0, d_{k}\right]$ during each execution of the algorithm. Note that

$$
\begin{aligned}
G_{k}(w) & =B\left(q_{k-1}+w\right)-B\left(q_{k-1}\right) \\
& =\int_{a_{k-1}}^{a_{k-1}+w} b(t) d t=-\int_{a_{k-1}}^{a_{k-1}+w} \frac{f^{\prime}(t)}{f(t)} d t \\
& =\ln \left[f\left(q_{k-1}\right) / f\left(q_{k-1}+w\right)\right] .
\end{aligned}
$$

Since only (b) is done on-line, the success of an algorithm would seem to depend only on the ability to evaluate $\ln f(x)$ rapidly. We thus see that having $f(x)=$ $C \exp (\varphi(x))$ (and hence a solution of an equation of type (1)) is of great practical advantage, but it is not essential in principle to the use of von Neumann's idea.

Computer Science Department

Stanford University

Stanford, California 94305

1. J. H. Ahrens \& U. Dieter, "Computer methods for sampling from the exponential and normal distributions," Comm. Assoc. Comput. Mach., v. 15, 1972.

2. U. DieTER \& J. Ahrens, "A combinatorial method for the generation of normally distributed random numbers." (To appear.)

3. JOHN VON NEUMANN, "Various techniques used in connection with random digits, in Monte Carlo Method, Appl. Math. Series, vol. 12, U. S. Nat. Bureau of Standards, 1951, pp. 36-38 (Summary written by George E. Forsythe); reprinted in John von Neumann, Collected Works. Vol. 5, Pergamon Press; Macmillan, New York, 1963, pp. 768-770. MR 28 \# 1104. 